\title{
Hollow system with fin. Transient Green function method combination for two hollow cylinders
}

\author{
Andris Buikis* \\ Institute of Mathematics and Computer Science, University of Latvia, Raina Blvd. 29, LV1459, Riga, LATVIA
}

\begin{abstract}
In this paper we develop mathematical model for three dimensional heat equation for the system with hollow wall and fin and construct its analytical solution for two hollow cylindrical sample. The method of solution is based on Green function method for one hollow cylinder. On the conjugation conditions between both hollow cylinders we construct solution for system wall with fin. As result we come to integral equation on the surface between both hollow cylinders. Solution is obtained in the form of second kind Fredholm integral equation. The generalizing of Green function method allows us to use Green function method for regular non-canonical domains.
\end{abstract}

\section{INTRODUCTION}

Systems with extended surfaces (fins, spines) are related to very different branches of technique. Usually their mathematical modeling is realized by one dimensional steady-state assumptions [1]-[3]. In our previous papers we have constructed various two and three dimensional analytical approximate and exact solutions [4 -25].

In [2] the so-called Murray - Gardner assumptions are formulated. Some of them are:

1) The heat flow in the fin and the temperature at any point on the fin remains constant with time;

4) The temperature of the medium surrounding the fin is uniform;

5) The fin width is small compared to its height, so the temperature gradients across the fin width may be neglected;

6) The temperature at the base of the fin is uniform;

7) The heat transferred through the outmost edge of the fin (the fin tip) is negligible compared to that through the lateral surfaces (faces) of the fin.

In this paper we obtain exact transient solution by the Green function method [11 -13]. We give up all of these Murray - Gardner assumptions.

We consider three-dimensional statement for nonhomogeneous equation with partly non-homogeneous boundary conditions.

In recent years, we have been able to generalize the Green's function method to areas, which consist of several canonical connected sub-areas, and thus we have obtained the exact solutions for the L-, T- and П-type areas [11 -13], [15,], 21, 23]. We have constructed two cylinders [17], and two-layer sphere [15].Cylinder was investigated in the paper [18].

\section{FORMULATION OF 3-D PROBLEM}

For this purpose instead of the time depended ordinary differential equation [1] - [3], we consider the following partial differential equation for the first cylinder:

$$
\begin{aligned}
& \frac{1}{a^{2}} \frac{\partial U}{\partial t}=\left[\frac{1}{r} \frac{\partial}{\partial r}\left(r \frac{\partial U}{\partial r}\right)+\frac{1}{r^{2}} \frac{\partial^{2} U}{\partial \varphi^{2}}+\frac{\partial^{2} U}{\partial z^{2}}\right]+ \\
& F(r, \varphi, z, t), r \in\left[R_{0}, R\right], \varphi \in[0,2 \pi], \\
& z \in[0, l], a^{2}=\frac{k}{c \rho} .
\end{aligned}
$$

Here $c$ is specific heat capacity, $k$ - heat conductivity coefficient, $\rho$ - density. For the second cylinder which can be of different material:

$$
\begin{aligned}
& \frac{1}{a_{0}^{2}} \frac{\partial U_{0}}{\partial t}=\left[\frac{1}{r} \frac{\partial}{\partial r}\left(r \frac{\partial U_{0}}{\partial r}\right)+\frac{1}{r^{2}} \frac{\partial^{2} U_{0}}{\partial \varphi^{2}}+\frac{\partial^{2} U_{0}}{\partial z^{2}}\right], \\
& r \in\left[R_{0}, R_{1}\right], \varphi \in[0,2 \pi], z \in\left[l, l_{0}\right], a_{0}^{2}=\frac{k_{0}}{c_{0} \rho_{0}} .
\end{aligned}
$$

We formulate the conjugations conditions as ideal thermal contact for $r \in\left[R_{0}, R_{1}\right]$.

$$
\begin{aligned}
& \left.U\right|_{z=l-0}=\left.U_{0}\right|_{z=l+0}, \\
& \left.k \frac{\partial U}{\partial z}\right|_{z=l-0}=\left.k_{0} \frac{\partial U_{0}}{\partial z}\right|_{z=l+0} .
\end{aligned}
$$

We assume that the outer diameter of second cylinder is smaller as for primary cylinder: $R_{1}<R$. The boundary conditions we assume partly homogeneous:

$$
\left.\left(\frac{\partial U}{\partial r}-k_{0} U\right)\right|_{r=R_{0}}=0, k_{0}=\frac{h}{k},
$$

\footnotetext{
* Corresponding author: buikis@latnet.lv
} 


$$
\begin{aligned}
& \left.\left(\frac{\partial U}{\partial r}+k_{0} U\right)\right|_{r=R}=g_{0}(\varphi, z, t), \\
& \left.\left(\frac{\partial U}{\partial z}-k_{0} U\right)\right|_{z=0}=g_{1}(r, \varphi, t), \\
& \left.\left(\frac{\partial U}{\partial z}+k_{1} U\right)\right|_{z=l, R_{1}<r<R}=g_{2}(r, \varphi, t), \\
& \left.\left(\frac{\partial U_{0}}{\partial r}-k_{2} U_{0}\right)\right|_{r=R_{0}}=0, k_{2}=\frac{h_{0}}{k_{0}}, \\
& \left.\left(\frac{\partial U_{0}}{\partial r}+k_{2} U_{0}\right)\right|_{r=R_{1}}=0, \\
& \left.\left(\frac{\partial U_{0}}{\partial z}+k_{3} U_{0}\right)\right|_{z=l_{1}}=g_{3}(r, \varphi, t) .
\end{aligned}
$$

Here $h, h_{0}$ is heat exchange coefficient. The initial conditions for the both cylinders are assumed in following form:

$$
\begin{aligned}
& \left.U\right|_{t=0}=U_{0}(r, \varphi, z), \\
& \left.U_{0}\right|_{t=0}=U_{00}(r, \varphi, z),
\end{aligned}
$$

For 3-D mathematical model it is important that solution in $\varphi-$ direction is continuous and smooth. These 2 conditions are important for the reduction of 3-D model to $2-\mathrm{D}$ model by conservative averaging method $[9,23]$ and [24]:

$$
\begin{gathered}
\left.U\right|_{\varphi=0}=\left.U\right|_{\varphi=2 \pi}, \\
\left.\frac{\partial U}{\partial \varphi}\right|_{\varphi=0}=\left.\frac{\partial U}{\partial \varphi}\right|_{\varphi=2 \pi} .
\end{gathered}
$$

\section{SOLUTION OF 3-D PROBLEM}

The Green function for two cylinders is not known. The new idea is to combine the two conjugations conditions in the form of third type boundary condition with unknown non-homogeneity in the right hand side.

\subsection{Solution for the wall}

As for the fin we combine the conjugations conditions (3), (4) for $R_{0}<r<R_{1}$ :

$$
\left.\left(\frac{\partial U}{\partial z}+k_{1} U\right)\right|_{z=l-0}=F_{0}(r, \varphi, t)
$$

$F_{0}(r, \varphi, t)=\left.\left(\frac{k_{0}}{k} \frac{\partial U_{0}}{\partial z}+k_{1} U_{0}\right)\right|_{z=l+0}$.

The Green function (1) is known; see [28]:

$G(r, \varphi, z, \xi, \eta, \zeta, t)=$

$G_{1}(r, \varphi, \xi, \eta, t) G_{2}(z, \zeta, t)$,

$G_{1}(r, \varphi, \xi, \eta, t)=\frac{1}{\pi} \sum_{n=0}^{\infty} \sum_{m=1}^{\infty} A_{n} \mu_{n m}^{2} \times$

$\frac{Z_{n}\left(\mu_{n m} r\right) Z_{n}\left(\mu_{n m} \xi\right)}{B_{n m}} \times$

$\frac{\cos [n(\varphi-\eta)] \sin \left(\lambda_{n m s} t\right)}{\lambda_{n m s}} \exp \left(-a_{\tau}^{2} \mu_{n m}^{2} t\right)$,

$B_{n m}=\left(k_{2}^{2} R+\mu_{n m}^{2} R^{2}-n^{2}\right) Z_{n}^{2}\left(\mu_{n m} R\right)-$

$\left(k_{2}^{2} R_{0}+\mu_{n m}^{2} R_{0}^{2}-n^{2}\right) Z_{n}^{2}\left(\mu_{n m} R_{0}\right)$,

$G_{2}(z, \zeta, t)=\sum_{s=1}^{\infty} \frac{h_{s}(z) h_{s}(\zeta)}{\left\|h_{s}\right\|^{2}} \exp \left(-a^{2} \lambda_{s}^{2} t\right)$.

The solution for three-dimensional problem for the wall (1) is in following form:

$U(r, \varphi, z, t)=H(r, \varphi, z, t)+a^{2} \int_{0}^{t} d \tau \times$

$\int_{0}^{2 \pi} d \varsigma \int_{R_{0}}^{R_{1}} F_{0}(\xi, \varsigma, \tau) G(r, \varphi, z, \xi, \varsigma, l, t-\tau) d \xi$

$+\int_{R_{0}}^{R_{1}} \xi d \xi \int_{0}^{2 \pi} d \varsigma \times$

$\int_{l}^{l_{1}} U_{0}(\xi, \eta, \varsigma) G(r, \varphi, z, \xi, \eta, \varsigma, t) d \eta$.

Here

$H(r, \varphi, z, t)=a^{2} R \times$

$\int_{0}^{t} d \tau \int_{0}^{2 \pi} d \eta \int_{0}^{l} g_{0}(\xi, \varsigma, \tau) G(r, \varphi, z, R, \eta, \varsigma, t-\tau) d \varsigma+$

$-a^{2} \times$

$\int_{0}^{t} d \tau \int_{0}^{2 \pi} d \varsigma \int_{R_{0}}^{R_{1}} g_{1}(\xi, \varsigma, \tau) \xi G(r, \varphi, z, \xi, \varsigma, 0, t-\tau) d \xi+$

$a^{2} \int_{0}^{t} d \tau \int_{0}^{2 \pi} d \varsigma \int_{R_{1}}^{R} g_{2}(\xi, \varsigma, \tau) G(r, \varphi, z, \xi, \varsigma, l, t-\tau) d \xi$.

Here $J_{n}(r), Y_{n}(r)-$ are Bessel's functions 


$$
\begin{aligned}
& Z_{n}\left(\mu_{n m} r\right)= \\
& {\left[\mu_{n m} J_{n}^{\prime}\left(\mu_{n m} R_{0}\right)-k_{2} J_{n}\left(\mu_{n m} R_{0}\right)\right] Y_{n}\left(\mu_{n m} r\right)} \\
& -\left[\mu_{n m} Y_{n}^{\prime}\left(\mu_{n m} R_{0}\right)-k_{2} Y_{n}\left(\mu_{n m} R_{0}\right)\right] J_{n}\left(\mu_{n m} r\right)
\end{aligned}
$$

and $\mu_{n m}$ are the positive roots of the transcendental equation:

$$
\begin{aligned}
& \frac{\left[\mu J_{n}^{\prime}\left(\mu R_{0}\right)-k_{0} J_{n}\left(\mu R_{0}\right)\right]}{\left[\mu J_{n}^{\prime}\left(\mu R_{1}\right)+k_{0} J_{n}\left(\mu R_{1}\right)\right]}= \\
& \frac{\left[\mu Y_{n}^{\prime}\left(\mu R_{0}\right)-k_{0} Y_{n}\left(\mu_{n m} R_{0}\right)\right]}{\left[\mu Y_{n}^{\prime}\left(\mu R_{1}\right)+k_{0} Y_{n}\left(\mu R_{1}\right)\right]} .
\end{aligned}
$$

For the second part of the Green function $G_{2}(z, \zeta, t)$ we have:

$$
\begin{aligned}
& h_{s}(z)=\cos \left(\lambda_{s} z\right)+\frac{k_{0}}{\lambda_{s}} \sin \left(\lambda_{s} z\right), \\
& \left\|h_{s}\right\|^{2}=\frac{k_{1}}{2 \lambda_{s}^{2}} \frac{\lambda_{s}^{2}+k_{0}^{2}}{\lambda_{s}^{2}+k_{1}^{2}}+\frac{k_{0}}{2 \lambda_{s}^{2}}+\frac{l}{2}\left(1+\frac{k_{0}^{2}}{\lambda_{s}^{2}}\right), \\
& A_{n}=\left\{\begin{array}{l}
1, \text { if } n=0, \\
2, \text { if } n>0 .
\end{array}\right.
\end{aligned}
$$

The eigenvalues $\beta_{s}$ are positive roots of the transcendental equations:

$$
\frac{\operatorname{tg}(\lambda l)}{\lambda}=\frac{k_{0}+k_{1}}{\lambda^{2}-k_{0} k_{1}} \text {. }
$$

The wall solution (18) contains the fin temperature and its derivative $F_{0}(\xi, \varsigma, \tau)$ in the formula (16). It means that we must solve the solution for the fin.

\subsection{Solution for the fin}

The combination of conjugations conditions (3), (4) gives such third type boundary condition :

$$
\begin{aligned}
& \left.\left(\frac{\partial U_{0}}{\partial z}-U_{0}\right)\right|_{z=l}=F(r, \varphi, t), \\
& F(r, \varphi, t)=\left.\left(\frac{k}{k_{0}} \frac{\partial U}{\partial z}-U\right)\right|_{z=l-0} .
\end{aligned}
$$

The solution in three-dimensional problem for the fin (2) is in following form:

$$
\begin{aligned}
& U_{0}(r, \varphi, z, t)=H_{0}(r, \varphi, z, t)+\int_{R_{0}}^{R_{1}} \xi d \xi \int_{0}^{2 \pi} d \eta \times \\
& \int_{l}^{l_{1}} U_{00}(\xi, \eta, \varsigma) G_{0}(r, \varphi, z, \xi, \eta, \varsigma, t) d \varsigma-a^{2} \int_{0}^{t} d \tau \times(24 \\
& \int_{0}^{2 \pi} d \eta \int_{R_{0}}^{R_{1}} F(\xi, \eta, \tau) \xi G_{0}(r, \varphi, z, \xi, \eta, l, t-\tau) d \xi .
\end{aligned}
$$

Here in the function we have collected all known boundary right hand side functions:

$$
\begin{aligned}
& H_{0}(r, \varphi, z, t)=a^{2} \times \\
& \int_{0}^{t} d \tau \int_{0}^{2 \pi} d \eta \int_{R_{0}}^{R_{1}} g_{3}(\xi, \eta, \tau) \xi G_{0}\left(r, \varphi, z, \xi, \eta, l_{1}, t-\tau\right) d \xi .
\end{aligned}
$$

The Green function is similar as (17) for initial-boundary problem for Klein-Gordon equation (2).It is known; see [27]:

$$
\begin{aligned}
& G_{0}(r, \varphi, z, \xi, \eta, \zeta, t)= \\
& G_{01}(r, \varphi, \xi, \eta, t) G_{02}(z, \zeta, t), \\
& G_{01}(r, \varphi, \xi, \eta, t)=\frac{1}{\pi} \sum_{n=0}^{\infty} \sum_{m=1}^{\infty} A_{n} \mu_{0 n m}^{2} \times \\
& \frac{Z_{n}\left(\mu_{0 n m} r\right) Z_{n}\left(\mu_{0 n m} \xi\right)}{B_{0 n m} \times} \\
& \frac{\cos [n(\varphi-\eta)] \sin \left(\lambda_{s} t\right)}{\lambda_{s}} \exp \left(-a_{\tau}^{2} \mu_{0 n m}^{2} t\right), \\
& B_{0 n m}=\left(k_{2}^{2} R_{1}+\mu_{0 n m}^{2} R_{1}^{2}-n^{2}\right) Z_{n}^{2}\left(\mu_{0 n m} R_{1}\right)- \\
& \left(k_{2}^{2} R_{0}+\mu_{0 n m}^{2} R_{0}^{2}-n^{2}\right) Z_{n}^{2}\left(\mu_{0 n m} R_{0}\right), \\
& G_{2}(z, \zeta, t)=\sum_{s=1}^{\infty} \frac{h_{0 s}(z) h_{0 s}(\zeta)}{\left\|h_{0 s}\right\|^{2}} \exp \left(-a_{0}^{2} \lambda_{s}^{2} t\right) .
\end{aligned}
$$

Here $\mu_{0 n m}$ are the positive roots of the transcendental equation:

$$
\begin{aligned}
& {\left[\mu J_{n}^{\prime}\left(\mu R_{0}\right)-k_{2} J_{n}\left(\mu R_{0}\right)\right] \times} \\
& {\left[\mu Y_{n}^{\prime}\left(\mu R_{1}\right)+k_{2} Y_{n}\left(\mu R_{1}\right)\right]=} \\
& {\left[\mu Y_{n}^{\prime}\left(\mu R_{0}\right)-k_{2} Y_{n}\left(\mu_{n m} R_{0}\right)\right] \times} \\
& {\left[\mu J_{n}^{\prime}\left(\mu R_{1}\right)+k_{2} J_{n}\left(\mu R_{1}\right)\right] .}
\end{aligned}
$$

For the second part of the Green function $G_{02}(z, \zeta, t)$ we have: 


$$
\begin{aligned}
& h_{0 s}(z)=\cos \left(\lambda_{0 s} z\right)+\frac{\sin \left(\lambda_{0 s} z\right)}{\lambda_{0 s}}, \\
& \left\|h_{0 s}\right\|^{2}=\frac{k_{3}}{2 \lambda_{0 s}^{2}} \frac{\lambda_{0 s}^{2}+1}{\lambda_{0 s}^{2}+k_{3}^{2}}+\frac{1}{2 \lambda_{0 s}^{2}}+\frac{l_{1}-l}{2}\left(1+\frac{1}{\lambda_{0 s}^{2}}\right), \\
& A_{n}=\left\{\begin{array}{l}
1, \text { if } n=0, \\
2, \text { if } n>0 .
\end{array}\right.
\end{aligned}
$$

The eigenvalues $\beta_{s}$ are positive roots of the transcendental equations:

$$
\frac{\operatorname{tg}\left[\lambda\left(l_{1}-l\right)\right]}{\lambda}=\frac{1+k_{3}}{\lambda^{2}-k_{3}} \text {. }
$$

\section{SOLUTION AS COMBINATION OF WALL AND FIN SOLUTIONS}

The solution in three-dimensional problem for the fin (24) is in following form:

$$
\begin{aligned}
& U_{0}(r, \varphi, z, t)= \\
& -\bar{H}_{0}(r, \varphi, z, t)-a^{2} \int_{0}^{t} d \tau \int_{0}^{2 \pi} d \eta \times \\
& \int_{R_{0}}^{R_{1}} F(r, \varphi, t) G_{0}(r, \varphi, z, \xi, \eta, l, t-\tau) d \xi, \\
& \bar{H}_{0}(r, \varphi, z, t)=H_{0}(r, \varphi, z, t)+\int_{R_{0}}^{R_{1}} \xi d \xi \int_{0}^{2 \pi} d \eta \times \\
& \int_{l}^{l_{1}} U_{00}(\xi, \eta, \varsigma) G_{0}(r, \varphi, z, \xi, \eta, \varsigma, t) d \varsigma .
\end{aligned}
$$

Unfortunately, the representation (27) is unusable as solution for the fin because of the unknown function $F(r, \varphi, t)$, i.e. temperature in the wall $U(r, \varphi, z=l, t)$ and derivative of temperature.

From equation (16) we can write the combination $F_{0}(r, \varphi, t)$ :

$F_{0}(r, \varphi, t)=-\hat{\bar{H}}_{0}(r, \varphi, t)-a^{2} \int_{0}^{t} d \tau \int_{0}^{2 \pi} d \eta \times$

$\int_{R_{0}}^{R_{1}} F(\xi, \eta, \tau) \hat{G}_{0}(r, \varphi, \xi, \eta, \varsigma, t-\tau) d \xi$.

Here

$$
\begin{aligned}
& \hat{\bar{H}}_{0}(r, \varphi, t)= \\
& \left.\left(\frac{k_{0}}{k} \frac{\partial}{\partial z} \bar{H}_{0}(r, \varphi, z, t)-\bar{H}_{0}(r, \varphi, z, t)\right)\right|_{z=l}, \\
& \hat{G}_{0}(r, \varphi, \xi, \eta, \varsigma, t)= \\
& {\left.\left[\frac{k_{0}}{k} \frac{\partial}{\partial z} G_{0}(r, \varphi, z, \xi, \eta, \varsigma, t)-G_{0}(r, \varphi, z, \xi, \eta, \varsigma, t)\right]\right|_{z=l} .}
\end{aligned}
$$

Similarly we do with equations (23) and (18):

$U(r, \varphi, z, t)=\bar{H}(r, \varphi, z, t)+a^{2} \int_{0}^{t} d \tau \times$

$\int_{0}^{2 \pi} d \varsigma \int_{R_{0}}^{R_{1}} F_{0}(\xi, \varsigma, \tau) G(r, \varphi, z, \xi, \varsigma, l, t-\tau) d \xi$,

$\bar{H}(r, \varphi, z, t)=H(r, \varphi, z, t)+\int_{R_{0}}^{R_{1}} \xi d \xi \int_{0}^{2 \pi} d \varsigma \times$

$\int_{l}^{l_{1}} U_{0}(\xi, \eta, \varsigma) G(r, \varphi, z, \xi, \eta, \varsigma, t) d \eta$.

From equation (23) we can write the combination $F(r, \varphi, t)$ :

$$
\begin{aligned}
& F(r, \varphi, t)=\hat{\bar{H}}(r, \varphi, t)+a^{2} \int_{0}^{t} d \tau \times \\
& \int_{0}^{2 \pi} d \eta \int_{R_{0}}^{R_{1}} F_{0}(\xi, \eta, \tau) \hat{G}(r, \varphi, l, \xi, \eta, l, t-\tau) d \xi .
\end{aligned}
$$

Here

$\hat{\bar{H}}(r, \varphi, t)=\left.\left[\frac{k}{k_{0}} \frac{\partial}{\partial z} \bar{H}(r, \varphi, t)-\bar{H}(r, \varphi, t)\right]\right|_{z=l}$,

$\hat{G}(r, \varphi, l, \xi, \varsigma, l, t)=$

$\left.\left[\frac{k}{k_{0}} \frac{\partial}{\partial z} G(r, \varphi, z, \xi, \varsigma, l, t)-G(r, \varphi, z, \xi, \varsigma, l, t)\right]\right|_{z=l}$

As the last step we substitute the combination $F_{0}(\xi, \eta, \tau)$ from $(28)$

$$
\begin{aligned}
& F_{0}(\xi, \eta, \tau)=-\hat{\bar{H}}_{0}(\xi, \eta, \tau)-a^{2} \int_{0}^{\tau} d \tau_{1} \int_{0}^{2 \pi} d \eta_{1} \times \\
& \int_{R_{0}}^{R_{1}} F\left(\xi, \eta, \tau_{1}\right) \hat{G}_{0}\left(\xi, \eta, \xi, \eta_{1}, \varsigma, \tau-\tau_{1}\right) d \xi_{1},
\end{aligned}
$$




$$
\begin{aligned}
& \text { in the equation } \\
& F(r, \varphi, t)=\hat{\bar{H}}(r, \varphi, t)+a^{2} \int_{0}^{t} d \tau \times \\
& \int_{0}^{2 \pi} d \eta \int_{R_{0}}^{R_{1}} F_{0}(\xi, \eta, \tau) \hat{G}(r, \varphi, l, \xi, \eta, l, t-\tau) d \xi .
\end{aligned}
$$

Finally we obtain following non-homogeneous Fredholm integral equation of 2 nd kind:

$$
\begin{aligned}
& F(r, \varphi, t)=\Phi(r, \varphi, t)+a^{2} \int_{0}^{t} d \tau \int_{0}^{\tau} d \tau_{1} \int_{0}^{2 \pi} d \eta_{1} \times \\
& \int_{0}^{2 \pi} d \eta \int_{R_{0}}^{R_{1}} F\left(\xi, \eta, \tau_{1}\right) \Gamma(r, \varphi, l, \xi, \eta, l, t-\tau) d \xi .
\end{aligned}
$$

Here

$$
\begin{aligned}
& \Gamma(r, \varphi, l, \xi, \eta, l, t)=\int_{R_{0}}^{R_{1}} \hat{G}(r, \varphi, l, \xi, \eta, l, t) d \xi \times \\
& \int_{R_{0}}^{R_{1}} \hat{G}_{0}\left(\xi, \eta, \xi_{1}, \eta_{1}, \varsigma, \tau-\tau_{1}\right) d \xi_{1}, \\
& \Phi(r, \varphi, t)=\hat{\bar{H}}(r, \varphi, t)-a^{2} \int_{0}^{t} d \tau \times \\
& \int_{0}^{2 \pi} d \eta \int_{R_{0}}^{R_{1}} \hat{\bar{H}}_{0}(\xi, \eta, \tau) \hat{G}(r, \varphi, l, \xi, \eta, l, t-\tau) d \xi .
\end{aligned}
$$

We finish our paper with the following remark. The problem with non-homogeneous environment temperatures (6), (7), (8) and (11) and its solution allow conjugating temperature field with hydrodynamic field.

\section{CONCLUSIONS}

We have constructed exact three-dimensional transient analytical solution for one element with hollow cylinder fin. The solution is obtained in the form of Fredholm integral equation of 2 nd kind and has continuous kernel. As the result we come to integral equation on the surface between both hollow cylinders. The generalizing of Green function method allows us to use Green function method for regular non-canonical domains.

This work has been supported by Latvian Council of Sciences (grant 623/2014).

\section{References}

1. Kern, D.Q, Kraus, A.D. Extended Surface Heat Transfer. - McGraw-Hill Book Company (1972)

2. Kraus, A.D., Analysis and evaluation of extended surface thermal systems. Hemisphere publishing Corporation (1982)
3. Manzoor, M., Heat Flow Through Extended Surface Heat Exchangers. Springer-Verlag: Berlin and New York (1984)

4. Buikis, A., Two-dimensional solution for heat transfer in a regular fin assembly. Latvian Journal of Physics and Technical Sciences, No. 5, pp. 51-58 (1995)

5. Buikis, A., Buike, M. Approximate analytical twodimensional solution for a longitudinal fin of rectangular profile. Acta Universitatis Latviensis, 612, pp.33-44 (1998)

6. Buikis A., Buike M. Closed two-dimensional solution for heat transfer in a periodical system with a fin. Proceedings of the Latvian Academy of Sciences. Section B, 52, No.5, pp. 218-222. (1998)

7. Buike M. Simulation of steady-state heat process for the rectangular fin-containing system, Mathematical Modelling and Analysis, 4, pp. 33-43 (1999)

8. Malik, M.Y., Wood, A.S., Buikis, A. An approximate analytical solution to a familiar conjugate heat transfer problem. International journal of Pure and Applied Mathematics, Vol.10, Nr.1, pp. 91-107. (2004)

9. Buike M., Buikis A. Approximate Solutions of Heat Conduction Problems in Multi- Dimensional Cylinder Type Domain by Conservative Averaging Method, Part 1. Proceedings of the 5th IASME/WSEAS Int. Conf. on Heat Transfer, Thermal Engineering and Environment, Vouliagmeni, Athens, August $25-27$, p. $15-20$, (2007).

10. Buike M., Buikis A. Several Intensive Steel Quenching Models for Rectangular Samples. Proceedings of NAUN/WSEAS International Conference on Fluid Mechanics and Heat \&Mass Transfer, Corfu Island, Greece, July 22-24. p. 88-93 (2010).

11. Buike M., Buikis A. Hyperbolic heat equation as mathematical model for steel quenching of L-shape samples, Part 1 (Direct Problem). Applied and Computational Mathematics. Proceedings of the 13th WSEAS International Conference on Applied Mathematics (MATH'08), Puerto De La Cruz, Tenerife, Canary Islands, Spain, December 15-17, WSEAS Press, p. 198-203, (2008).

12. Bobinska T., Buike M., Buikis A. Hyperbolic Heat Equation as Mathematical Model for Steel Quenching of L-Shape Samples, Part 2 (Inverse Problem). Proceedings of 5th IASME/WSEAS International Conference on Continuum Mechanics (CM'10), University of Cambridge, UK, February 23-25, p. 21-26, (2010).

13. Bobinska T., Buike M., Buikis A. Hyperbolic Heat Equation as Mathematical Model for Steel Quenching of L-and T-Shape Samples, Direct and Inverse Problems. Transactions of Heat and Mass Transfer. Vol.5, Issue 3, July 2010. p. 63-72.

14. Blomkalna S., Buikis A. Heat conduction problem for double-layered ball. Progress in Industrial Mathematics at ECMI 2012. Springer, p. 417-426, (2014) 
15. Bobinska T., Buike M., Buikis A. Comparing solutions of hyperbolic and parabolic heat conduction equations for L-shape samples. Recent Advances in Fluid Mechanics and Heat@Mass Transfer. Proceedings of the 9th IASME/WSEAS International Conference on THE'11. Florence, Italy, August 23-25, p. 384-389 (2011).

16. M. Buike, A. Buikis, R. Vilums. One-Dimensional Intensive Steel Quenching Models. Recent Advances in Mechanical Engineering. Proceedings of the 5th International Conference on Fluid Mechanics and Heat \& Mass Transfer. Lisbon, Portugal, October 30- November 1, p. 54-62, (2014).

17. Piliksere A., Buikis A. Analytical solution for intensive quenching of cylindrical sample. Proceedings of 6th International Scientific Colloquium "Modelling for Material Processing", Riga, September 16-17, p. 181-186, (2010).

18. Piliksere A., Buike M., Buikis A. Steel quenching process as hyperbolic heat equation for cylinder. Proceedings of 6th Baltic Heat Conference BHTC2011, ISBN 978-952-15-2640-4 (CD-ROM), (2011).

19. Buike M., Buikis. Exact Transient Solution for System with Rectangular Fin, Proceedings of the 5th WSEAS Int. Conf. on Heat and Mass Transfer, Acapulco, Mexico, January 25-27, 2008, pp. 25-30.

20. Lencmane M. and Buikis A. Analytical solution of a two-dimensional double-fin assembly. Recent Advances in Fluid Mechanics and Heat \& Mass Transfer, Proceedings of the 9th IASME/WSEAS International Conference on Heat Transfer, Thermal Engineering and Environment (HTE'11), Florence, Italy, August 23 - 25, pp. 396 - 401, (2011).

21. Lencmane M. and Buikis A. Analytical solution for steady stable and transient heat process in a doublefin assembly. International Journal of Mathematical Models and Methods in Applied Sciences 6, No. 1. p.81-89, (2012).

22. Lencmane M., Buikis A. Some new mathematical models for the Transient Hot Strip method with thin interlayer. Proc. of the 10th WSEAS Int. Conf. on Heat Transfer, Thermal engineering and environment (HTE '12), WSEAS Pres, p. 283-288, (2012).

23. Buikis A., Buike M., Ulanova N., Solutions for the Transient Heat Transfer in Periodical Systems with Fins. Numerical Mathematics and Mathematical Problems of Mechanics. Ukrainian Mathematical congress - 2001, Section 8, Kiev, 2002, pp.15-23 (in Russian).

24. Buikis A. Conservative averaging as an approximate method for solution of some direct and inverse heat transfer problems. Advanced Computational Methods in Heat Transfer, IX. WIT Press, p. 311-320, (2006).

25. Vilums R., Buikis A. Conservative averaging method for partial differential equations with discontinuous coefficients. WSEAS Transactions on Heat and Mass Transfer. 1, Issue 4, p. 383-390, (2006).
26. Roach G.F. Green's Functions. Cambridge University Press, (1999).

27. Carslaw H.S., Jaeger C.J. Conduction of Heat in Solids. Oxford, Clarendon Press (1959).

28. Polyanin A.D. Handbook of Linear Partial Differential Equations for Engineers and Scientists. Chapman \& Hall/CRC, (Russian edition, 2001). (2002). 